\title{
A chaotic prediction algorithm using a new cost function
}

\author{
$\mathrm{Bu}$ Yun \\ School of Electrical and Information Engineering \\ Xihua University \\ Chengdu, China \\ e-mail: buyun_uestc@yahoo.cn
}

\author{
Kang Wan Xin \\ School of Electrical and Information Engineering \\ Xihua University \\ Chengdu, China \\ e-mail: buyun_1226@163.com
}

\begin{abstract}
The traditional cost function, minimization mean square prediction error is not a proper cost function in chaotic series prediction, for many chaotic signals are non-Gaussian distributions. Then we present using minimization error negentropy as new cost function, and derive the nonlinear approximation method. In simulation, the algorithm shows an enhanced performance to a common two order Volterra prediction.
\end{abstract}

Keywords-Chaotic time series, density function, cost function, negentropy

\section{INTRODUCTION}

As well known, dynamic systems are sensitive to initial conditions, so the future trajectories are difficult to track. But the wide potential applications, such as weather forecast and stock price prediction inspired people to have been exploring new methods to find out a feasible model that can describe the future values of a chaotic system. At present, much effective methods have been proposed. Local models are very powerful if the data base is large enough with low noise level. The representative linear algorithm is autoregressive model ${ }^{[1,2]}$ :

$$
\hat{x}(n+T)=\alpha_{0}+\sum_{i=1}^{m} \alpha_{i} x(n-(d-1) \tau)
$$

where $\alpha_{i}$ is the weight value, and $\mathrm{T}$ is the predicting time length, if $\mathrm{T}=1$, then (1) is a one step prediction, otherwise, if $\mathrm{T}>1$, it is a multi-step ahead prediction.

The local linear prediction method search for the closest neighbors and employ their images as the prediction, thus the scheme is almost model free. But the algorithm show poor performance if the data base is not large enough, or a compute cost is a significant consideration. Then advanced methods and global modeling attracted more attention. People want to build a valid functional form that can model a dynamic system on the whole attractor. A popular strategy is:

$$
\hat{x}(n+T)=\sum \alpha_{i} f_{i}(X(n))
$$

where $f_{i}(x)$ is a basis function, $X(n)$ is the input vector, and $\alpha_{i}$ is the weight value.

In the equation (2), basis functions often are polynomial, radial basis functions, or fuzzy functions. Besides, neural networks ${ }^{[3-9]}$ are other popular modeling tools. All the mentioned approaches are different from each others, but in training, they all get their coefficients on the minimization of the one-step mean squared prediction error. The cost function is suitable only in the special case of Gaussian distribution of prediction errors. But this is always true because a chaotic signal is often non-Gaussian distribution, and if the prediction accuracy is not high enough, a prediction algorithm will yield non-Gaussian prediction errors. So the cost function of minimization the one-step mean squared prediction error will lead to an over fitting problem, in some case even deterioration algorithm performance.

\section{MINIMIZATION THE ERROR NEGENTROPY}

A one-step prediction algorithm can write as

$$
\hat{x}(n+1)=F(\bar{X}(n))
$$

Then the prediction error is

$$
e(n+1)=x(n+1)-\hat{x}(n+1)=x(n+1)-F(\bar{X}(n))
$$

Generally, we suppose the prediction errors are normal distribution, which is the one with the highest entropy in all distributions. This implies the prediction errors are random and unpredictable. If a prediction algorithm is not perfect, the errors will be difference in entropy with Gaussian distribution, and the difference will can be measured by negentropy:

$$
J(e(n))=H(y)-H(e(n))
$$

Where $H(y)$ is the differential entropy of the Gaussian density with the same mean and variance as $e(n)$. The differential entropy is defined as

$$
H(v)=-\int f(\varepsilon) \log f(\varepsilon) d \varepsilon
$$

Where $f(v)$ is the density function of $v$.

Because the normal distribution has the highest entropy, the negentropy is always nonnegative. Thus using minimization the negentropy as the cost function is more suitable than minimization the one-step mean squared prediction error. In independent component analysis (ICA), the negentropy can be approximated by ${ }^{[10]}$ :

$$
J(y) \propto[E(G(y))-E(G(v))]^{2}
$$

Where $\mathrm{G}(\mathrm{x})$ is a non-quadratic function, $\mathrm{v}$ is a standard Gaussian variable.

If the prediction algorithm is the formal (2), the prediction error will be:

$$
e(n)=x(n)-\sum \alpha_{i} f_{i}(X(n-1))
$$


Then the partial derivative of the prediction error negentropy is

$$
\begin{aligned}
\frac{\partial J(e(n))}{\partial \alpha} & \propto\left\{E(G(e(n))-E(G(v))\} \bullet E\left\{\frac{\partial}{\partial \alpha} G(e(n))\right\}\right. \\
& =\gamma \bullet E\left\{G^{\prime}(e(n)) \bullet\left(\sum f_{i}(X(n-1))\right)\right\}
\end{aligned}
$$

Where $\gamma=E(G(e(n))-E(G(v))$. From the equation (9), we can derive random gradient algorithm:

$$
\Delta \alpha(n)=\gamma G^{\prime}(e(n)) \bullet\left(\sum f_{i}(X(n-1))\right.
$$

III.

The new cost function was introduced to an adaptive prediction algorithm, Volterra method to test its performance:

$$
\begin{aligned}
\hat{y}(k+1) & =a+\sum_{i_{1}=0}^{L-1} a_{i_{1}} y\left(k-i_{1}\right)+\sum_{i_{1} \leq i_{2}} a_{i_{1} i_{2}} y\left(k-i_{1}\right) y\left(k-i_{2}\right) \\
& +\ldots+\sum_{i_{1} \leq i_{2} \leq . \leq i_{p}} a_{i_{1} i_{2} \ldots i_{p}} y\left(k-i_{1}\right) y\left(k-i_{2}\right) \ldots y\left(k-i_{p}\right)+\ldots
\end{aligned}
$$

In simulation, the order of Volterra filter was assigned to 2. We used the criterion, mean square error (MSE) to monitor the accuracy of the prediction model:

$$
M S E=10 \log _{10}\left[\frac{\sum_{i=1}^{K} e^{2}(i)}{\sum_{i=1}^{K} x^{2}(i)}\right]
$$

A common chaotic system, coupled Logistic system was used as tested system, defined as:

$$
\begin{aligned}
& x(n+1)=4 a x(n)(1-x(n))+r x(n) y(n), \\
& y(n+1)=4 a y(n)(1-y(n))+r x(n) y(n)
\end{aligned}
$$

where $a=0.7, r=0.64$.

The benchmark time series contain 5500 data produced by the equation (13), where the former 5000 data were used to achieve the coefficients, and the residuals were test data. All the data were normalized in advance:

$$
\begin{aligned}
& A=(\max (x)+\min (x)) / 2 \\
& B=(\max (x)-\min (x)) / 2 \\
& y=(x-A) / B
\end{aligned}
$$

The nonlinear function that applied in (10) is

$$
G^{\prime}(x)=x \exp \left(-x^{2} / 2\right)
$$

The simulation results are shown in figure 1 and 2 . The former manifests the predicted values and the actual ones in test set, and the mean MSE is $-15.12 \mathrm{~dB}$ for ten different initial values. In fact, the result is better than the presented algorithms like the methods presented in [8] and [9]. Figure (2) tells the prediction errors distribution. From figure (2), we find the errors are close to Gaussian distribution, but the difference is also obviously. Mainly, the plot is not symmetric, it slightly skews to left. The difference shows that the algorithm can not exploit enough information; there are some things that can be improved.

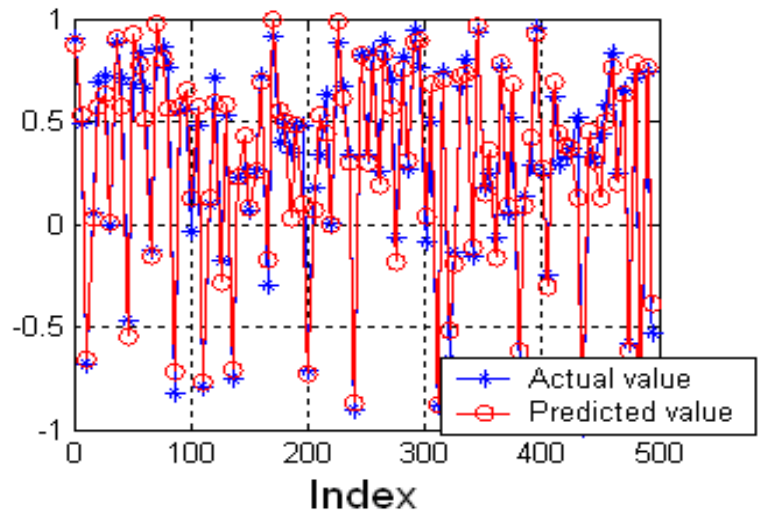

Fig.1 Prediction result

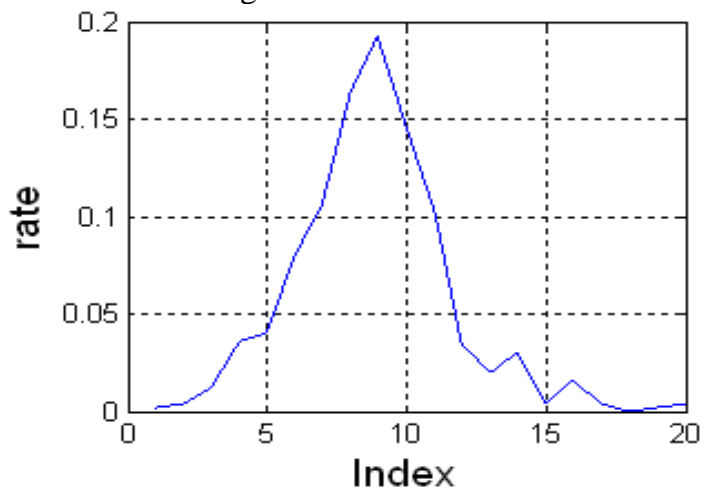

Fig.2 Prediction errors density

\section{CONCLUSIONS}

We analysis and propose a new cost function that used in prediction algorithm. The new cost function is derived from negentropy, which measures the difference between prediction errors and Gaussian signals. In prediction, we minimized the difference to achieve prediction method. This algorithm is tested and proved its effective to improve the performance of a common method.

\section{ACKNOWLEDGMENTS}

This work is supported by the research foundation of the education bureau of $\mathrm{Si}$ Chuan Province and Xihua University through Grants No. 12ZB132 and No.Z1120944.

\section{REFERENCES}

[1] Kantz H. and Schreiber T. Nonlinear time series analysis. Cambridge university press, 2004.

[2] Casdagli M. Nonlinear prediction of chaotic time series. Physica D, 1989, 35(3): 335-356

[3] Casdagli M. Chaos and deterministic versus stochastic nonlinear modeling. Journal of the Royal Statistical Society. Series B (Methodological), Vol. 54, No. 2 (1992), pp. 303-328

[4] Gong Xiaofeng, and C. H. Lai, Phys. Rev. E, Improvement of the local prediction of chaotic time series, Vol. 60, No. 5, pp. 5463-5468, Nov. 1999 
[5] Min Han, Jianhui Xi, Shiguo Xu, and Fu-Liang Yin, Prediction of chaotic time series based on the recurrent predictor neural network, IEEE Trans. on Sig. Proc. Vol. 52, No. 2, pp. 3409-3416, Dec. 2004

[6] Zhang Jia-Shu, and Xiao Xian-Ci, Predicting chaotic time series using recurrent neural network, Chin. Phys. Lett. Vol. 17, No. 2, pp. 88-90, 2000

[7] Vamsi K.Yadavalli, Rahul K.Dholes, Sanjeev S. Tambe, and B. D. Kulkarni, Obtaining functional form for chaotic time series evolution using genetic algorithm, Chaos, Vol. 9, No. 3, pp. 789-794, Sept. 1999
[8] BU Yun, WEN Guang-Jun, ZHOU Xiao-Jia, ZHANG Qiang. A novel adaptive predictor for chaotic time series. Chin. Phys. Lett. 2009, vol.26, No.10: 100502

[9] Zhang J. S, Xiao X.C. Prediction of chaotic time series by using adaptive higher-order nonlinear fourier infrared filter. Acta Physica Sinica, vol.49, No.7, 2000: 1221-07

[10] Aapo H. Juha K. Erkki O. Independent component analysis, John Wiley \& Sons, Inc. 2001 